\title{
Suppression of vortex shedding from a pantograph head using vortex generator-type plasma actuators
}

\author{
Haruhiko GEJIMA*, Ryo TAKINAMI*, Koji FUKAGATA*, Takeshi MITSUMOJI**, Takeshi SUEKI** and \\ Mitsuru IKEDA** \\ ${ }^{*}$ Department of Mechanical Engineering, Keio University \\ 3-14-1 Hiyoshi, Kohoku-ku, Yokohama, Kanagawa 223-8522, Japan \\ E-mail: fukagata@mech.keio.ac.jp \\ ${ }^{* *}$ Railway Technical Research Institute \\ 2-8-38 Hikari-cho, Kokubunji-shi, Tokyo 185-8540, Japan
}

Received 17 February 2015

\begin{abstract}
A vortex generator (VG) is one of the most successful devices used for suppression of flow separation. A conventional VG is a passive control device, so that it may suffer from an inherent drag penalty. As an alternative, we use a dielectric-barrier-discharge plasma actuator (PA) as a VG. Since few studies have been made on such a vortex generator-type plasma actuator (VG-PA), many questions still remain open, e.g., the effectiveness and the performance of the VG-PA for suppression of vortex shedding from a bluff body. In the present study, we experimentally evaluate the performance of the VG-PA by applying it to a pantograph head model of high-speed trains. The Reynolds number based on the freestream velocity and the width of the pantograph head model is 7200 . The spanwise velocity induced by the VG-PA is about $33 \%$ of the freestream velocity. The flow field is measured by using particle image velocimetry. The present results show that the vortex shedding from a pantograph head model can significantly be suppressed by the VG-PA, which amounts to about $60 \%$ reduction in the transverse velocity fluctuations. It is also shown that a burst discharge control significantly improves the control effect in the single-side configuration, in which the vortex shedding is not much suppressed in the continuous mode.
\end{abstract}

Key words : Flow separation control, Plasma actuator, Vortex generator, Pantograph head, Burst signal input

\section{Introduction}

Vortex shedding is often observed in flows around two- or three-dimensional bluff bodies. It is caused by flow separation, and it generates noise and vibrations. Those problematic issues have motivated us to suppress the flow separation for many years. In recent years, use of vortex generators (VGs) has been considered one of the most effective methods for suppression of flow separation. Vortex generators prevent the flow separation by creating an array of streamwise vortices close to the surface of a body. The streamwise vortices enhance the mixing of momentum between the boundary layer and the freestream; high-momentum fluid in the outer flow is entrained into the near wall region, enabling the flow near the wall to gain the energy to withstand the adverse pressure gradient. A vane-type VG (Lin, 2002) is the most widely used device. It consists of a row of small plates sticking out normal to the body at a small incidence to the freestream. It creates a pressure difference between the plate surfaces, which leads to a longitudinal vortex from the tip. Since probably the first vane-type VG was tested by Taylor (1947), it has been used in many practical applications such as commercial aircrafts.

The success of the vane-type VG is based on the fact that it creates streamwise vortices with distinct organization and longevity (Bushnell, 1992), which effectively work to suppress the flow separation. Furthermore, it has a simple structure and can easily be applied to a wide range of applications. However, a vane-type VG cannot cope with various velocity conditions and it suffers from an inherent drag penalty. The drag penalty is always present even when the control effect is not required. Therefore, less intrusive methods to generate streamwise vortices are desired to resolve such problems. 


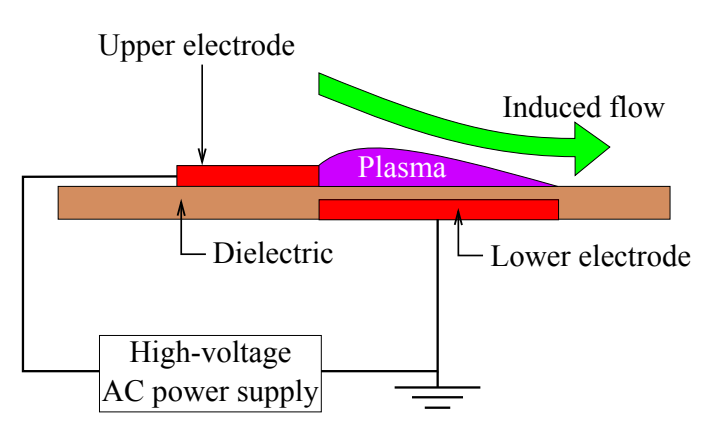

Fig. 1 The typical construction of a PA.

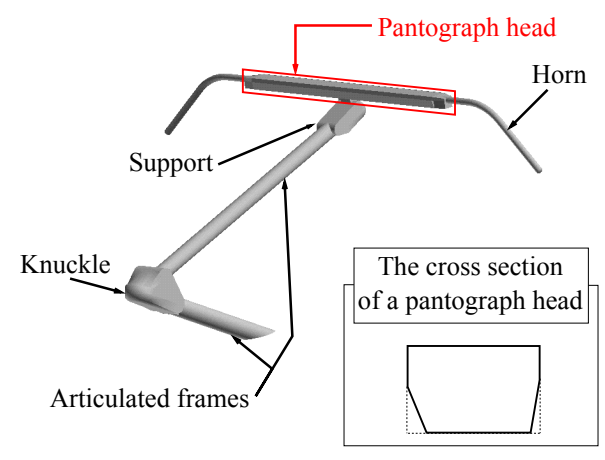

Fig. 3 A pantograph head on the train body.

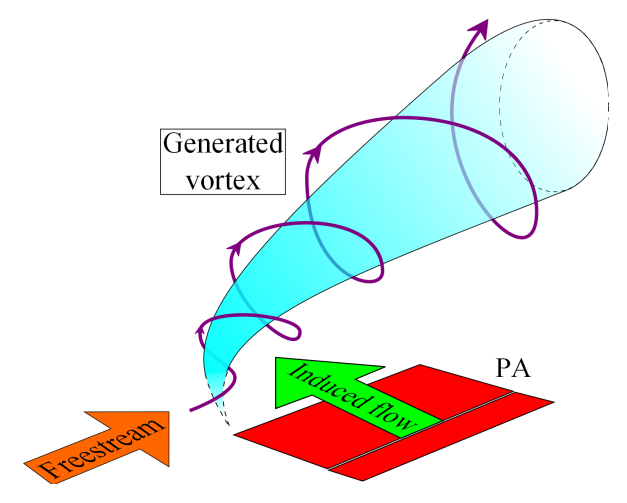

Fig. 2 A vortex generator-type plasma actuator (VG-PA).

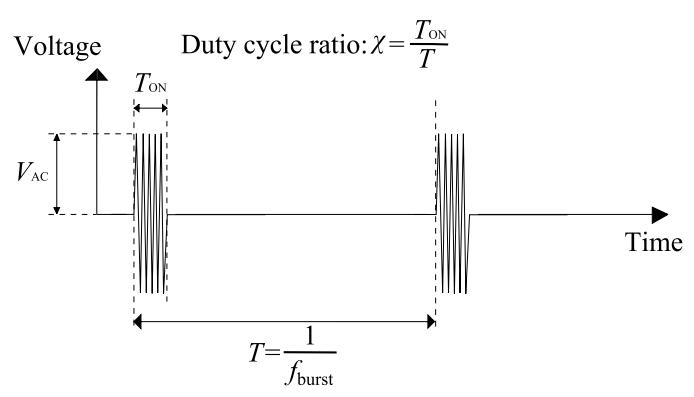

Fig. 4 The burst signal.

Recently, application of a dielectric-barrier-discharge plasma actuator (PA) to flow separation control has attracted increasing attentions. The PA is a purely electric device; it creates plasma close to the aerodynamic surface and induces a jet flow directed from the upper electrode to the lower electrode (Roth and Dai, 2006; Moreau, 2007; Roy and Wang, 2008). A typical PA consists of two electrodes separated by a dielectric layer, as shown in Fig. 1. Plasma actuators have successfully been applied to boundary layers (Corke et al., 2010) and flows around bluff bodies (Jukes and Choi, 2009a, 2009b). In many studies, PAs have been used to induce a flow in the streamwise direction (Jukes and Choi, 2009a, 2009b; Nati et al., 2013). In these cases, the velocity of the induced flow should have the same order of magnitude as the freestream velocity, otherwise the induced flow is too weak to directly interfere with the freestream. This is especially problematic in high-speed flows appearing in real applications. Two approaches have been proposed to overcome this problem: one is to use a burst signal for the driving voltage signal (hereafter referred to as the burst mode) (Asada and Fujii, 2012), the other is to use the PA as a device to generate vortices, i.e., a vortex generator-type plasma actuator (VG-PA), as shown in Fig. 2 (Jukes and Choi, 2013).

The VG-PA is expected to work as a VG without unnecessary drag penalty; it is also expected to efficiently suppress separation at a lower induced velocity as compared to the PA inducing a streamwise flow. Jukes and Choi (2012) experimentally assessed the performance of a single VG-PA in a laminar boundary layer. Using a cross-stream particle image velocimetry (PIV), they studied the development of the streamwise vortex and the dependency of the induced velocity on the electrode length and the yaw angle. They also investigated the mechanism of vortex generation (Jukes and Choi, 2013). In terms of practical applications, however, only few studies using VG-PAs have been reported (Jukes et al., 2013): questions on the effectiveness of VG-PAs for suppression of vortex shedding from a bluff body still remain open.

In the present study, we experimentally evaluate the effect of VG-PAs for suppression of vortex shedding. For the evaluation, a flow around a pantograph head (i.e., an electric current collector on the top of a pantograph) model, as shown in Fig. 3 (Mitsumoji et al., 2013), is chosen. The pantograph head is chosen because it is one of the major aerodynamic noise sources of the Shinkansen bullet train in Japan; in fact, the operation speed of the Shinkansen is mainly constrained by the wayside-noise regulation (Makino et al., 1998). Two different modes of the driving signal are considered: the continuous mode, in which the AC signal is continuously applied to PAs, and the burst mode, in which the applied voltage is intermittently turned on and off as shown in Fig. 4. The burst signal has a good affinity with the PA and can improve the flow control effect of PA (Sidorenko et al., 2013). We investigate the effect of the duty cycle ratio, $\chi$, and the burst frequency, $f_{\text {burst }}$, and compare the results with those in the continuous mode. 
(a)

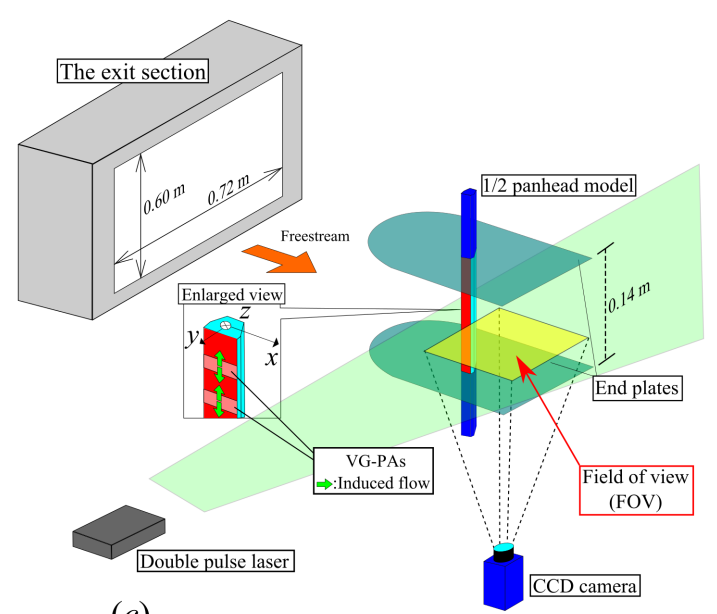

(c) (b)
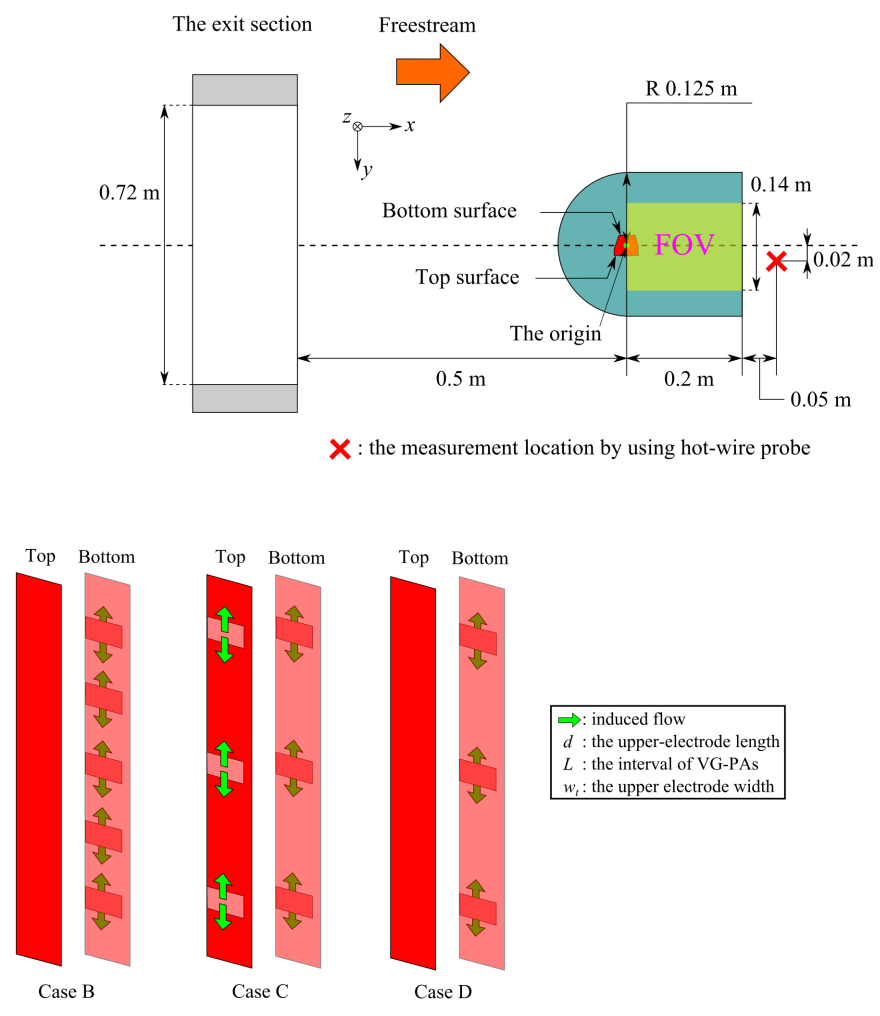

Fig. 5 Schematic of the experimental setup: $(a)$ overview of the instruments; $(b)$ overhead view of the experimental setup; $(c)$ the geometric configurations of the VG-PAs.

Table 1 Specification the VG-PA.

\begin{tabular}{cc}
\hline Parameter & Value \\
\hline Thickness of dielectric layer & $0.25 \mathrm{~mm}$ \\
Electrode length & $22.5 \mathrm{~mm}$ \\
Upper electrode width & $2.25 \mathrm{~mm}$ \\
Applied AC voltage $\left(V_{\mathrm{pp}}\right)$ & $16 \mathrm{kV}$ \\
Applied AC frequency & $4.0 \mathrm{kHz}$ \\
Induced velocity $\left(u_{\mathrm{PA}}\right)$ & $1.6 \mathrm{~m} / \mathrm{s}$ \\
\hline
\end{tabular}

\section{Experimental procedure}

\subsection{Experimental setup}

Experiments were conducted in the open-return wind tunnel at Railway Technical Research Institute (RTRI), Japan. The area of the exit section is $0.60 \times 0.72 \mathrm{~m}^{2}$. The freestream velocity was fixed at $U_{\infty}=4.8 \mathrm{~m} / \mathrm{s}$ with the turbulence intensity of $0.55 \%$. The experimental equipments were set as illustrated in Figs. $5(a)$ and $(b)$. A pantograph head model, whose width is $D_{p}=22.5 \mathrm{~mm}$, was vertically immersed in the test section. The top surface $(y>0)$ and the bottom surface $(y<0)$ were defined by taking the center of the pantograph head model as the origin of the coordinates. As shown in Fig. 5 (c), four configurations of VG-PAs were considered. In Cases A and C, VG-PAs are installed on both (i.e., top and bottom) surfaces; while in Cases B and D, VG-PAs are on the bottom surface only. It is worth noting that Cases B and D are preferable in the practical applications, because the top surface of the actual pantograph head should not be modified so that it can contact with the overhead contact wire. The difference between Cases A and B and Cases C and D is the spacing between VG-PAs: $L / D_{p}=1$ in Cases A and C, $L / D_{p}=2$ in Cases B and D.

A $0.25 \mathrm{~mm}$-thick polyimide tape (Kapton ${ }^{\circledR}$ ) and copper tapes were used as a dielectric layer and the electrodes, respectively. The specification of a single VG-PA is shown in Table 1. All VG-PAs were connected in a parallel circuit and simultaneously driven by a $16 \mathrm{kV}_{\mathrm{pp}} \mathrm{AC}$ voltage signal at a frequency of $4 \mathrm{kHz}$ generated by a power supply (PSIPG1040F, KI Tech.), which results in an induced velocity of $u_{\mathrm{PA}}=1.6 \mathrm{~m} / \mathrm{s}$ for each actuator.

The development of the streamwise vorticity field due to the VG-PAs is illustrated in Fig. 6. The red rectangles in the 
(a)

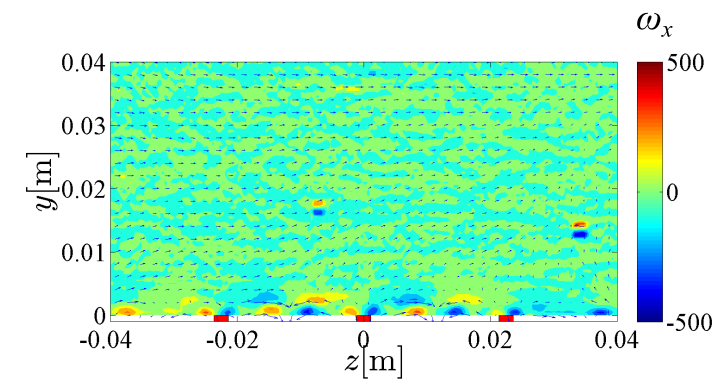

(b)

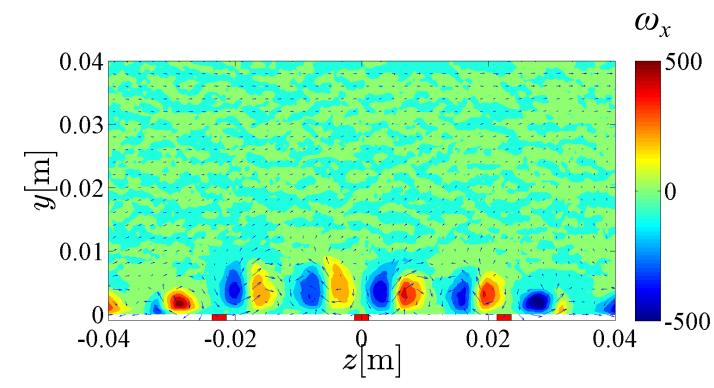

(c)

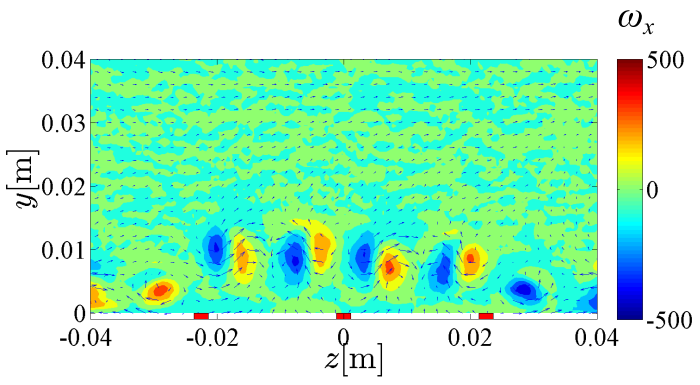

(d)

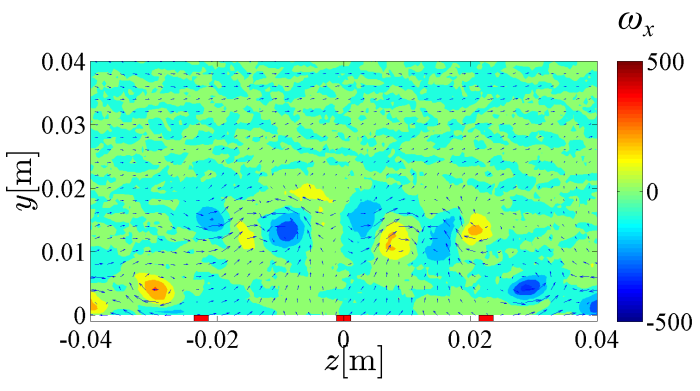

(e)

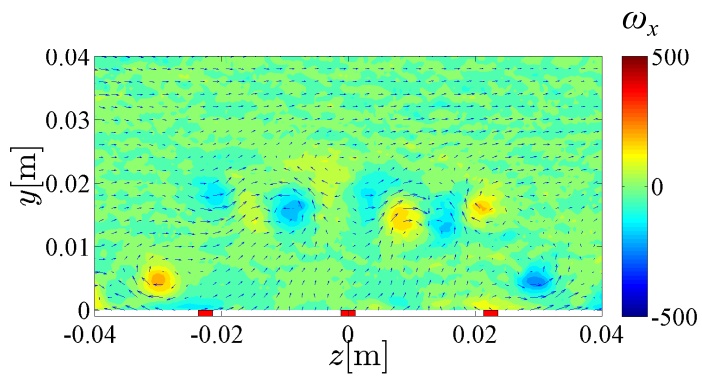

Fig. 6 Cross-sectional streamwise vorticity $\left(\omega_{x}\right)$ fields at different streamwise locations: $(a) x / D_{p}=0$ (at the origin); (b) $x / D_{p}=1 ;(c) x / D_{p}=2 ;(d) x / D_{p}=3 ;(e) x / D_{p}=4$.

figures indicate the upper electrodes of the VG-PAs. Note that the vorticity field presented here is just for confirmation of the primary effect of VG-PAs, and is measured on the flat plate with the VG-PAs in a wind tunnel at Keio University, which is different from that used for the main experiment. The Reynolds number is $\mathrm{Re}=1900$ and the induced velocity is $u_{\mathrm{PA}} / U_{\infty}=0.43$. At the origin (i.e., $x / D_{p}=0$ ), spanwise wall jets are found to be induced by the VG-PAs as illustrated in Fig. $6(a)$. At $x / D_{p}=1$, some pairs of longitudinal vortices are discerned near the upper electrodes as shown in Fig. $6(b)$. In the downstream side of the VG-PAs, the vortices are lifted up (Fig. $6(c)$ ) to eventually form more circular vortices, whose diameter is about a half of the actuator spacing (Figs. $6(d)-(e)$ ). From these observations, the VG-PAs are considered to generate longitudinal vortices to enhance the momentum exchange.

\subsection{Experimental conditions}

The experimental conditions are summarized in Table 2. The freestream velocity of $U_{\infty}=4.8 \mathrm{~m} / \mathrm{s}$ results in the Reynolds number of $\operatorname{Re}=U_{\infty} D_{p} / v=7200$. The vortex shedding frequency was investigated by using a hot-wire probe (Kanomax, IHW100) by measuring the freestream velocity at $(x, y, z)=(250 \mathrm{~mm},-20 \mathrm{~mm}, 0 \mathrm{~mm})$, as illustrated in Fig. 5 (b). In order to investigate the influence on the end plates to the vortex shedding, the vortex shedding frequency was measured without and with the end plates. The power spectrum density (PSD) of the measured streamwise velocity in the cylinder wake in these two cases are plotted in Fig. 7. The peak value becomes higher with the end plates, which implies a stronger two-dimensionality of the flow field as compared to that in the case without the end plates. From the peak in the PSD, the frequency of the vortex shedding from the pantograph head model is found to be $f_{\text {shed }}=23.8 \mathrm{~Hz}$, which results 
Table 2 Experimental conditions.

\begin{tabular}{cc}
\hline Parameter & Value \\
\hline Freestream velocity, $U_{\infty}$ & $4.8 \mathrm{~m} / \mathrm{s}$ \\
Width of pantograph head model, $D_{p}$ & $22.5 \mathrm{~mm}$ \\
Reynolds number, Re & 7200 \\
Strouhal number, St & 0.11 \\
Intervals of VG-PAs, $L$ & $22.5 \mathrm{~mm}, 45.0 \mathrm{~mm}$ \\
\hline
\end{tabular}

Table 3 Parameters for the burst signal.

\begin{tabular}{cc}
\hline Parameter & Value \\
\hline Duty cycle ratio, $\chi$ & $0.1-0.5$ \\
Burst frequency, $f_{\text {burst }}$ & $21.5 \mathrm{~Hz}-172.0 \mathrm{~Hz}$ \\
\hline
\end{tabular}

Table 4 Parameters for PIV.

\begin{tabular}{cc}
\hline Setup parameter & Value \\
\hline Number of pixels in captured image $(x \times y)$ & $1600 \times 1200$ pixels \\
Pulse separation & $2.0 \times 10^{-4} \mathrm{~s}$ \\
Objective focal length & $50 \mathrm{~mm}$ \\
Fieid of view (FOV) & $0.20 \times 0.14 \mathrm{~m}^{2}$ \\
Digital imaging resolution & $8.0 \times 10^{3} \mathrm{~m} /$ pixel \\
Interrogation window (first step) & $32 \times 32 \mathrm{pixels}$ \\
Interrogation window (second step) & $16 \times 16$ pixels \\
Number of vectors per field $(x \times y)$ & $99 \times 74$ \\
Number of recordings & 400 \\
\hline
\end{tabular}

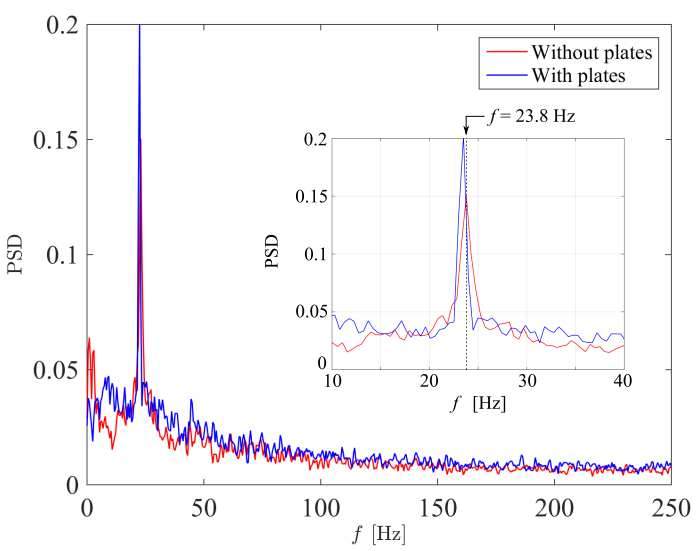

Fig. 7 The power spectrum density (PSD) of the measured streamwise velocity in the cylinder wake.

in the Strouhal number of $\mathrm{St}=D_{p} f_{\text {shed }} / U_{\infty}=0.11$ in accordance with the previous study (Mitsumoji et al., 2013). In the burst mode, the burst frequency, $f_{\text {burst }}$, was set close to integer multiples of $f_{\text {shed. }}$. The duty cycle ratio, $\chi$, was also varied from 0.1 to 0.5 as shown in Table 3 .

The flow field was measured by using a two-dimensional two-component PIV system. The system consists of a $200 \mathrm{~mJ}$ double-pulse Nd:YAG laser (New Wave Research, Gemini-PIV200), a $1600 \times 1200$ pixel CCD camera (PCO, PCO1600) and a PC. Dioctyl sebacate (DOS) seeding particles of nominally $1 \mu \mathrm{m}$ in diameter were introduced downstream of the wind tunnel, then circulated back to the flow around the pantograph head model. Although the ozone generated by VG-PAs and the high electric field strength close to the VG-PAs may be considered to affect the seeding particles, such an effect was not observed in the present experiment. The CCD camera was mounted under the test section as shown in Fig. 5. The field of view (FOV) through a $50 \mathrm{~mm}$ macro lens is $0.14 \mathrm{~m} \times 0.20 \mathrm{~m}$ in $x \times y$ directions around the central cross-section of the pantograph head model. Since plasma actuators were intermittently placed along the pantograph model (i.e., in $z$ direction), there would be a significant variation in the flow field and vortex shedding behavior at different $z$ locations. Therefore, the present experiments were carried out at different $z$ locations; $z=0 \mathrm{~mm}$ (on a VG-PA) and $z=11.25 \mathrm{~mm}$ (between two VG-PAs). The other parameters used in the PIV measurement are shown in Table 4.

The post-processing of PIV was performed using a commercial software, Koncerto II ver. 0.3.0.86 (Seika Digital Image Corp., Japan). First, the velocity vectors are computed on a $32 \times 32$ pixel grid; subsequently, they are refined on a $16 \times 16$ pixel grid using a recursive cross-correlation technique, by which 7326 vectors are obtained in each frame. This processing scheme resulted in less than $3.6 \%$ erroneous vectors. The image calibration was performed by using a calibration target; the conversion was typically $0.125 \mathrm{~mm} /$ pixel with $\pm 4.1 \%$ variation over the measurement area.

\section{Results and discussion}

\subsection{Uncontrolled case}

Figure 8 shows the mean centerline velocity, $U_{c}$, the profiles of the mean velocity, $U$, and the root-mean-square (RMS) transverse velocity fluctuations, $v_{\mathrm{rms}}$, at different downstream locations. The present results are compared with the results for a square cylinder obtained by a Laser Doppler Velocimetry (LDV) by Lyn et al. (1995) at $R e=21400$ and a hot-wire measurement by Saha et al. (2000) at $R e=8700$, because no result on the flow around a body with exacty the same shape is available in literature. Note that Saha et al. (2000) also made a measurement at $R e=17625$ and found no significant dependency of the nondimensionalized velocity profiles on the Reynolds number. As can be noticed from the figures, the flow around the pantograph head model is essentially similar to that around a square cylinder. The flow separation results in a broad wake and vortex sheddings, as illustrated by the contours of $U$ and $v_{r m s}$ in Fig. 9. Although 
Gejima, Takinami, Fukagata, Mitsumoji, Sueki and Ikeda, Journal of Fluid Science and Technology, Vol.10, No.1 (2015)
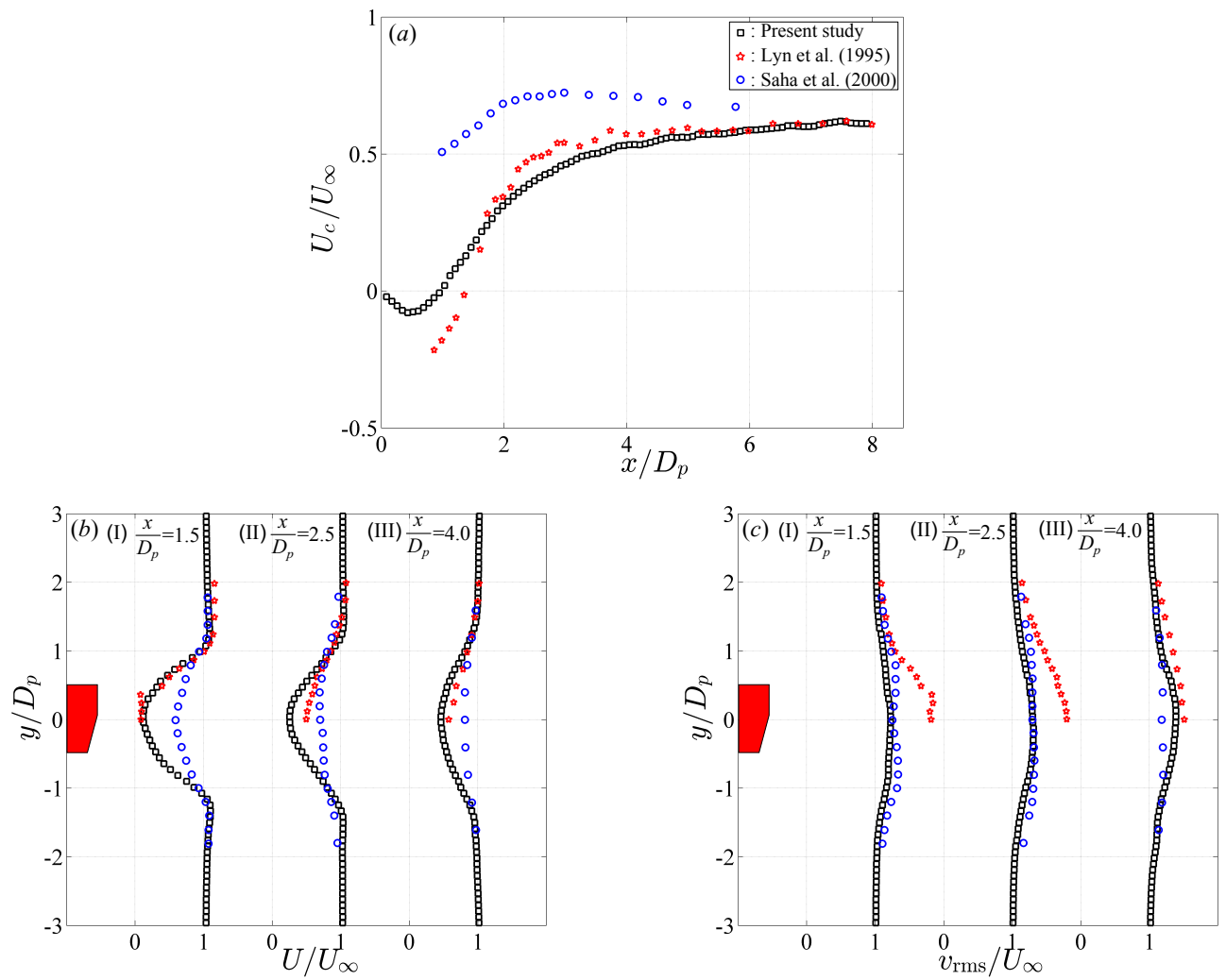

Fig. 8 Comparison with flow around a square cylinder: $(a)$ centerline velocity, $U_{c} ;(b)$ mean velocity profile, $U$; (c) RMS of transverse velocity fluctuations, $v_{\mathrm{rms}}$.

(a)

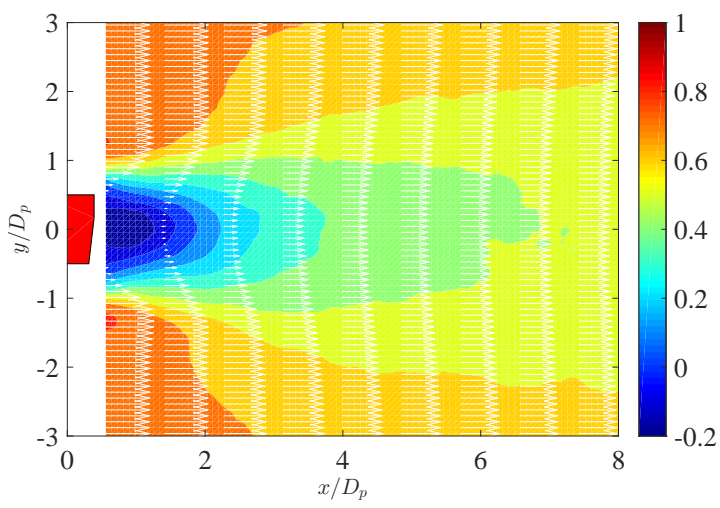

(b)

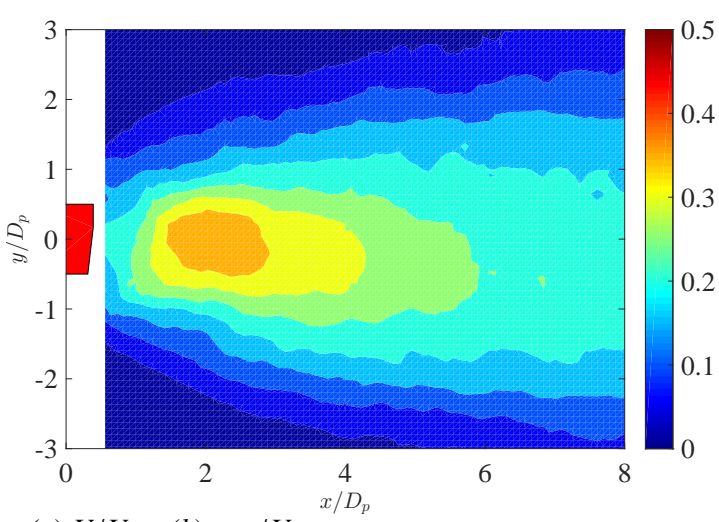

quantitative differences can be observed among the compared data, considering the discrepancies found even between the different experiments of a square cylinder, one may state that the physical phenomena are qualitatively similar.

\subsection{Overview of control effects}

Figure 10 shows the distributions of $U$ and $v_{\text {rms }}$ in Cases A-D in the continuous mode. The velocity fluctuations are significantly suppressed in Cases A and C (i.e., the cases where PAs were installed on both sides: hereafter referred to as double-side configurations). In these cases, the wake is observed to be thinner and longer, which indicates suppressions of both the flow separation on the body and the mixing in the shear layers. In Cases B and D (i.e., the cases where PAs were installed on the bottom surface only: single-side configurations), in contrast, the distributions of $U$ and $v_{\text {rms }}$ are not much changed from those in the uncontrolled case.

As a measure of suppression of vortex shedding, we define a reduction rate of the transverse velocity fluctuations, $R_{v}$, as

$$
R_{v}=\frac{\mathcal{V}_{0}-\mathcal{V}}{\mathcal{V}_{0}} \times 100 \% .
$$


Gejima, Takinami, Fukagata, Mitsumoji, Sueki and Ikeda,

Journal of Fluid Science and Technology, Vol.10, No.1 (2015)

(a)

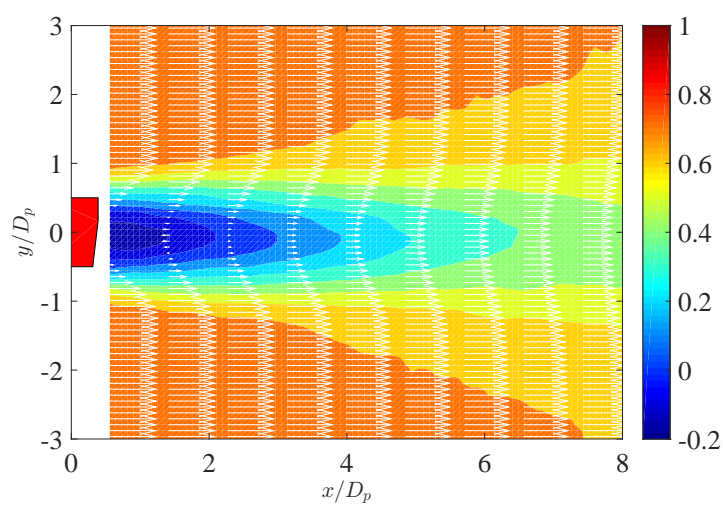

(b)

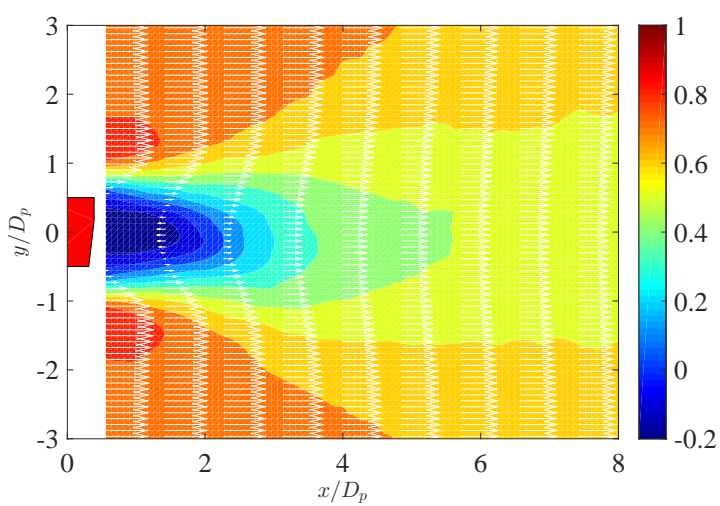

(c)

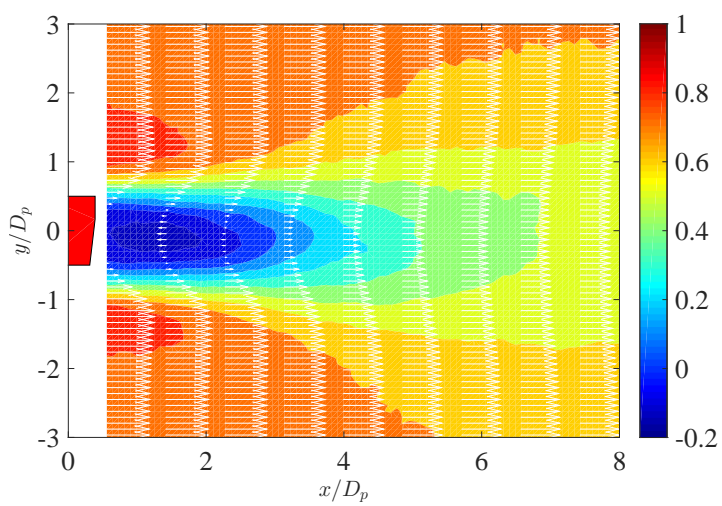

(d)

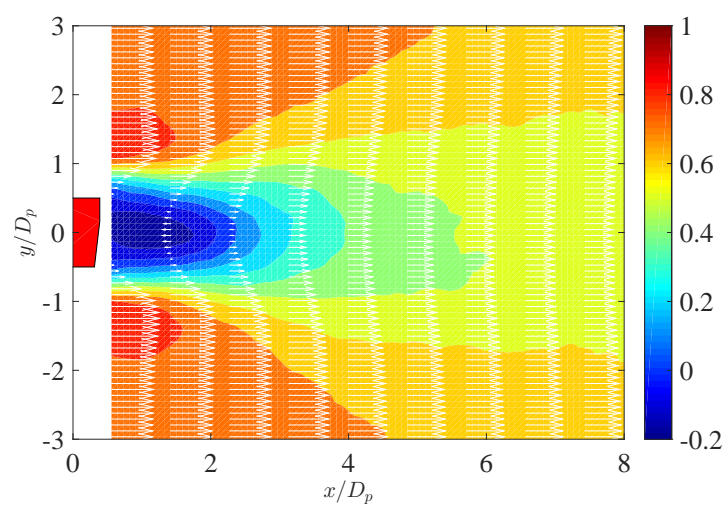

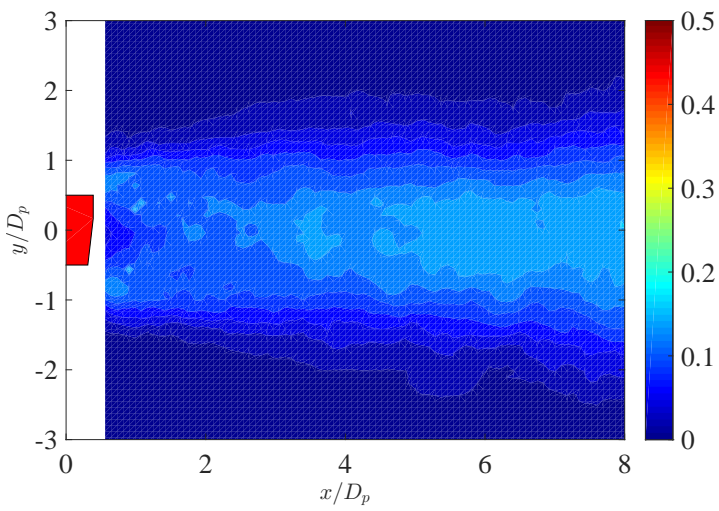
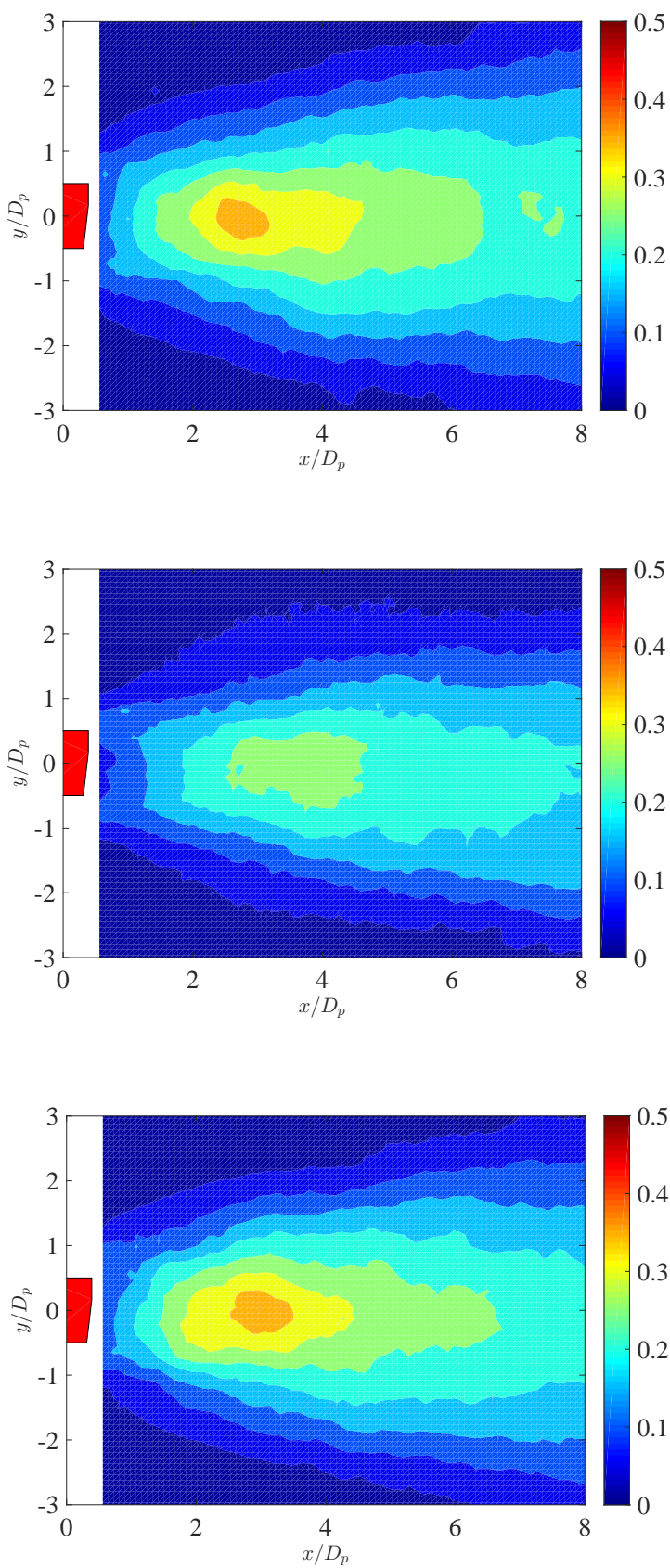

Fig. 10 Statistics in the continuous mode: (left) $U / U_{\infty}$; (right) $v_{\mathrm{rms}} / U_{\infty}$ : (a) Case A; (b) Case B; (c) Case C; $(d)$ Case D. 
Table 5 Reduction rate of the maximum $v_{\mathrm{rms}}, R_{v}[\%]$.

\begin{tabular}{|c|c|c|}
\hline & Continuous mode & $\begin{array}{c}\text { Burst mode } \\
\left(f_{\text {burst }} / f_{\text {shed }}=3.6, \chi=0.5\right)\end{array}$ \\
\hline Case A & 57 & 60 \\
\hline Case B & 5 & 30 \\
\hline Case $\mathrm{C}$ & 26 & 45 \\
\hline Case D & 5 & 18 \\
\hline
\end{tabular}

(a)

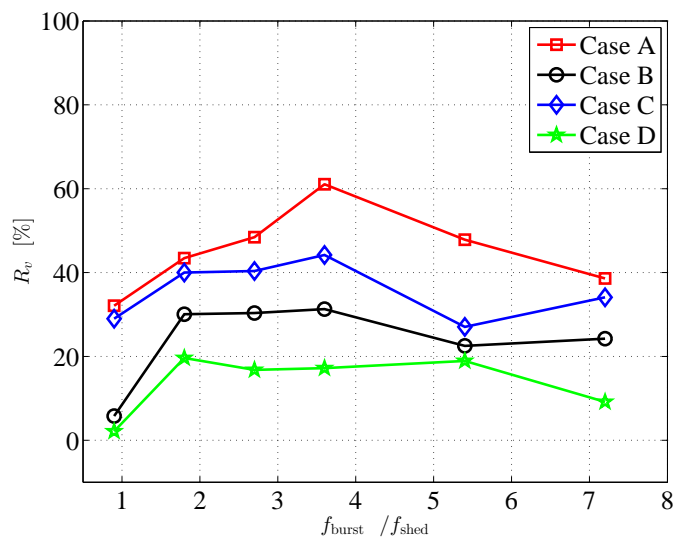

(b)

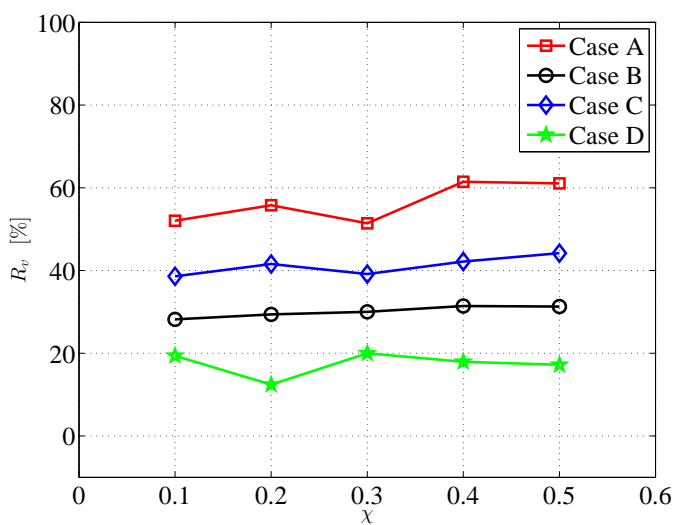

Fig. 11 Dependency of $R_{v}$ on $(a)$ the burst frequency, $f_{\text {burst }}(\chi=0.5)$; $(b)$ the duty cycle ratio, $\chi\left(f_{\text {burst }} / f_{\text {shed }}=3.6\right)$.

Here, $\mathcal{V}$ denotes the maximum value of $v_{\text {rms }}$ and the subscript of 0 denotes the uncontrolled case. The evaluated values of $R_{v}$ are tabulated in Table 5. The reduction rate is much higher in Cases $\mathrm{A}$ and C (double-side configurations) than Cases B and D (single-side configurations). Within the double-side configurations, Case A $\left(L / D_{p}=1\right)$ shows better performance than Case $\mathrm{C}\left(L / D_{p}=2\right)$.

Table 5 also indicates that the burst mode is more effective than the continuous mode. Figures $11(a)$ and $(b)$ show $R_{v}$ as functions of the burst frequency, $f_{\text {burst }}$, at the duty cycle ratio of $\chi=0.5$, and $R_{v}$ as functions of $\chi$ at $f_{\text {burst }} / f_{\text {shed }}=3.6$, respectively. In all cases, $R_{v}$ takes the maximum values around $f_{\text {burst }} / f_{\text {shed }}=3.6$, while it is less sensitive to $\chi$. More importantly, the burst mode greatly enhances the effect in the single-side configurations (Cases B and D), which are more suitable for practical applications.

\subsection{Comparison between the continuous mode and the burst mode}

In order to compare the effects of the continuous mode and the burst mode in more detail, we focus on the statistics obtained in Cases A and B.

Figures $12(a)$ and $(b)$ show the distributions of $U$ and $v_{\text {rms }}$ in the burst mode $\left(f_{\text {burst }} / f_{\text {shed }}=3.6, \chi=0.5\right)$ of Case A. The wake is observed to be narrower than that in the uncontrolled case, and the fluctuations are suppressed similarly to those in the continuous mode (cf. Fig. $10(a)$ ). Thus, the basic effect in this case is considered similar to that in the continuous mode, i.e., suppression of flow separation at its early stage, although the detailed mechanism of separation suppression may be different. Dependencies of $U_{c}$ and $v_{\mathrm{rms}}$ on the burst frequency are depicted in Figs. $12(c)$ and $(d)$, respectively. At $f_{\text {burst }} / f_{\text {shed }}=3.6$, both profiles are similar to those in the continuous mode. At a lower frequency, $f_{\text {burst }} / f_{\text {shed }}=0.9$, the profile of $U_{c}$ becomes similar to that in the uncontrolled case despite the shortened recirculation region, while suppression of $v_{\mathrm{rms}}$ is still found in the downstream wake. At a higher frequency, $f_{\text {burst }} / f_{\text {shed }}=5.4$, in contrast, the mean velocity recovers to the level of the uncontrolled flow more quickly than that at $f_{\text {burst }} / f_{\text {shed }}=3.6$ despite the elongated recirculation region. The fluctuation, $v_{\mathrm{rms}}$, is suppressed similarly to the continuous mode in the near wake, but it keeps on increasing up to $x / D_{p}=4$ to result in a level similar to the case of $f_{\text {burst }} / f_{\text {shed }}=0.9$. In summary, the optimal burst frequency in Case $\mathrm{A}$ is $f_{\text {burst }} / f_{\text {shed }}=3.6$. At a lower frequency, the disturbance by the VG-PA is too weak to have a sufficient mixing in the near wake. At a higher frequency, mixing in the near wake is sufficient, but a too strong disturbance is likely to cause an additional instability in the far wake to lead to a quicker recovery.

Figures $13(a)$ and $(b)$ show the distributions of $U$ and $v_{\text {rms }}$ in the burst mode $\left(f_{\text {burst }} / f_{\text {shed }}=3.6, \chi=0.5\right)$ of Case B. In Case B, the burst mode is more efficient than the continuous mode (cf. Fig. $10(b)$ ): the wake becomes much narrower than that in the continuous mode, and the velocity fluctuations are more clearly suppressed. Dependencies of $U_{c}$ and $v_{\text {rms }}$ on the burst frequency are shown in Figs. $13(c)$ and $(d)$. At a lower frequency, $f_{\text {burst }} / f_{\text {shed }}=0.9$, profiles are similar 
Gejima, Takinami, Fukagata, Mitsumoji, Sueki and Ikeda, Journal of Fluid Science and Technology, Vol.10, No.1 (2015)

(a)

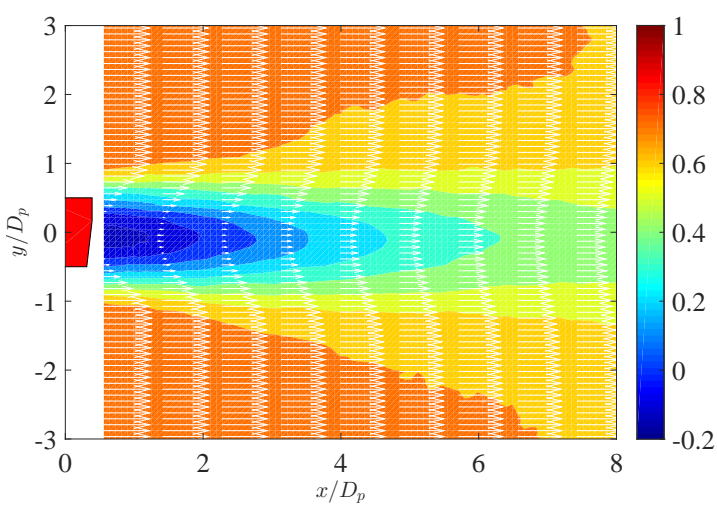

(c)

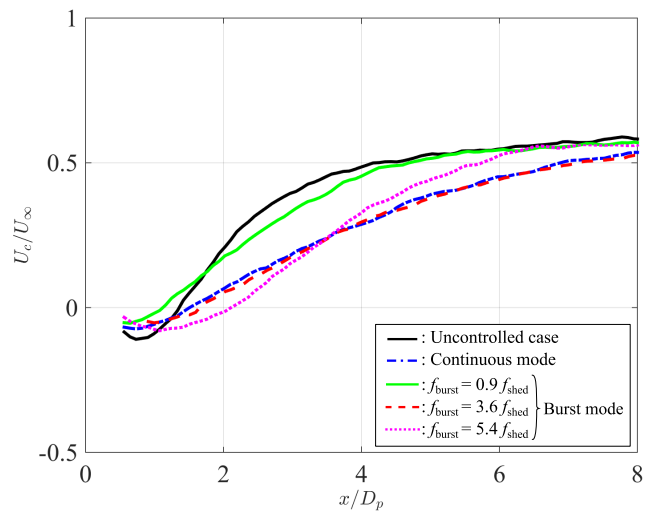

(b)

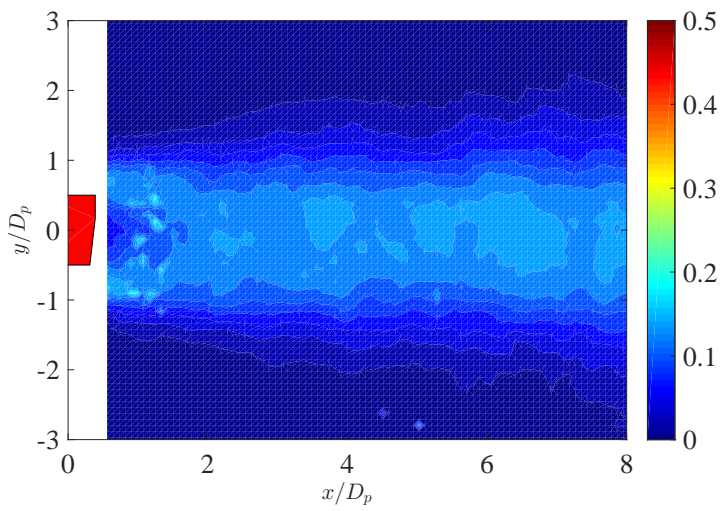

(d)

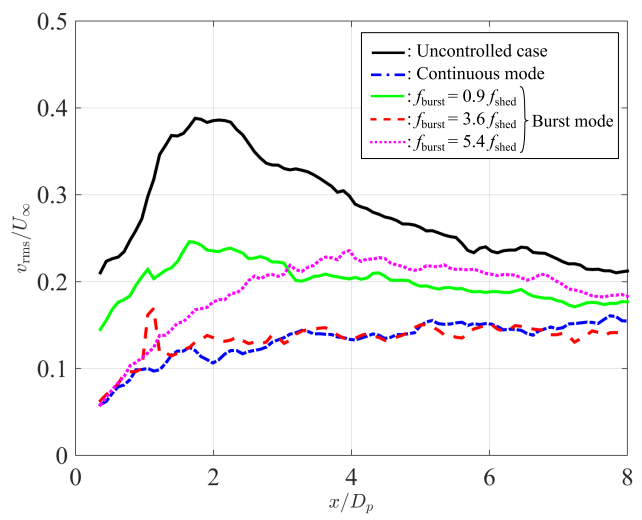

Fig. 12 Statistics in Case A at $z=0 \mathrm{~mm}:(a) U / U_{\infty}$ in the burst mode $\left(f_{\text {burst }} / f_{\text {shed }}=3.6\right)$; $(b) v_{\mathrm{rms}} / U_{\infty}$ in the burst mode $\left(f_{\text {burst }} / f_{\text {shed }}=3.6\right) ;(c) U_{c} / U_{\infty} ;(d) v_{\text {rms }} / U_{\infty}$ on the centerline. In all burst mode cases, $\chi=0.5$.

(a)

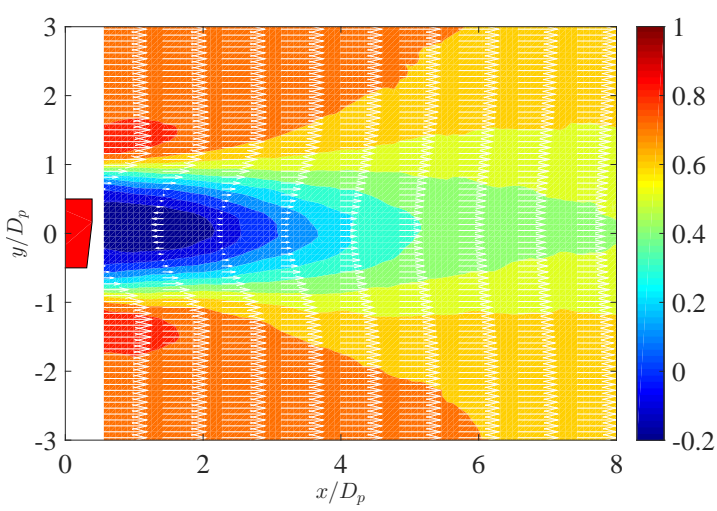

(c)

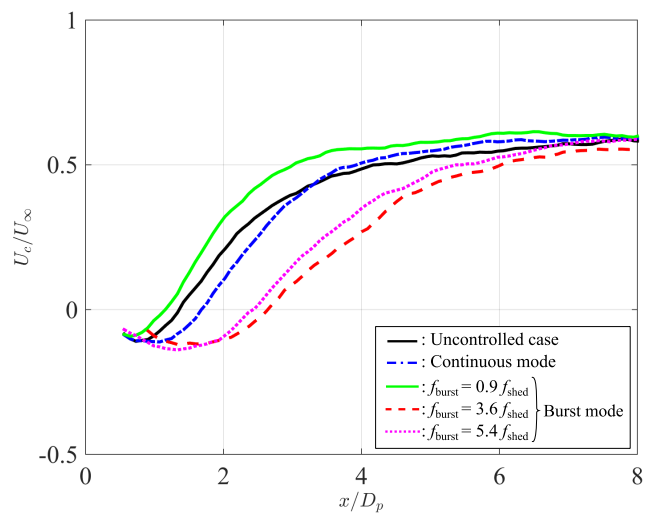

(b)

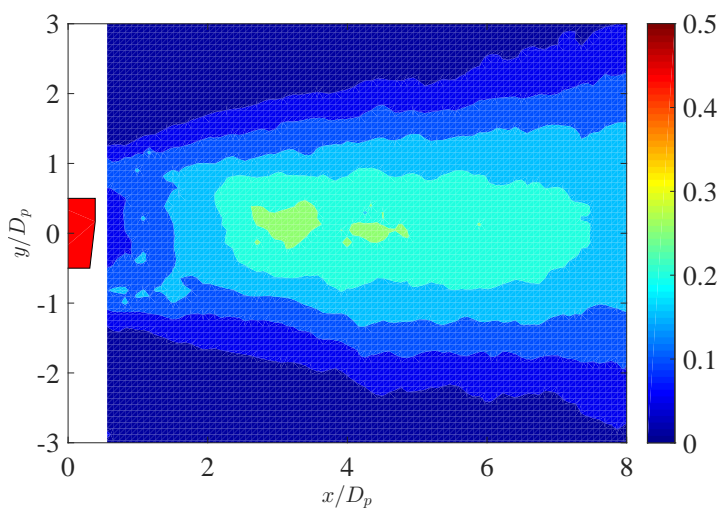

(d)

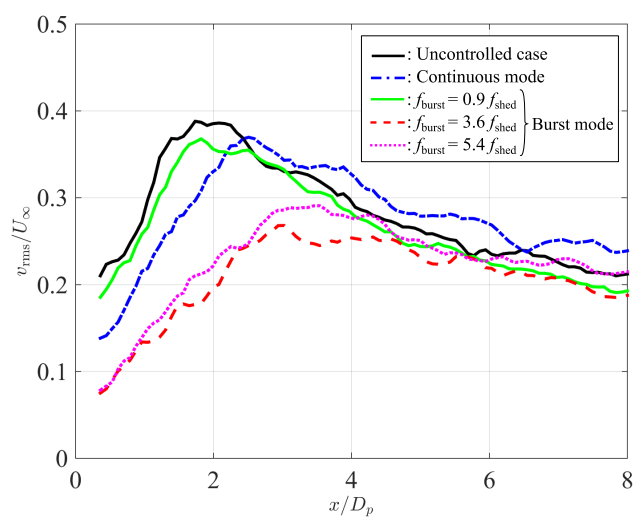

Fig. 13 Statistics in Case B at $z=0 \mathrm{~mm}:(a) U / U_{\infty}$ in the burst mode $\left(f_{\text {burst }} / f_{\text {shed }}=3.6\right) ;(b) v_{\text {rms }} / U_{\infty}$ in the burst mode $\left(f_{\text {burst }} / f_{\text {shed }}=3.6\right) ;(c) U_{c} / U_{\infty} ;(d) v_{\text {rms }} / U_{\infty}$ on the centerline. In all burst mode cases, $\chi=0.5$.

to those in the uncontrolled case and the case of continuous mode (in which a significant effect was not observed). At $f_{\text {burst }} / f_{\text {shed }}=3.6$ and 5.4, the recirculation region is found to be much elongated as compared to the uncontrolled case and 
(a)

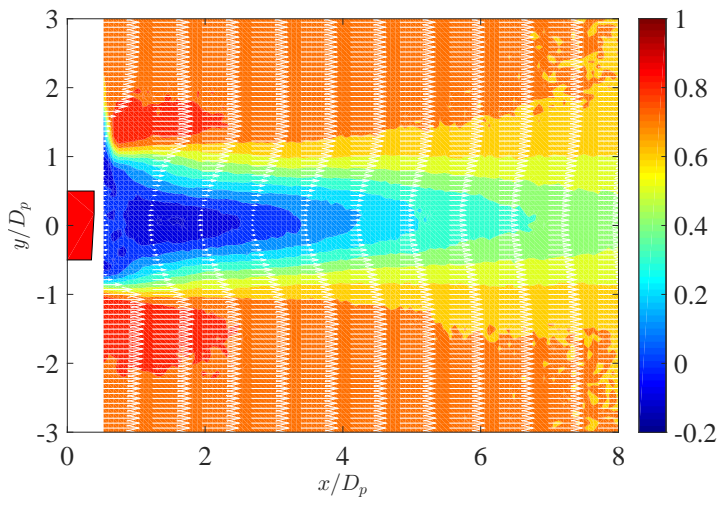

(c)

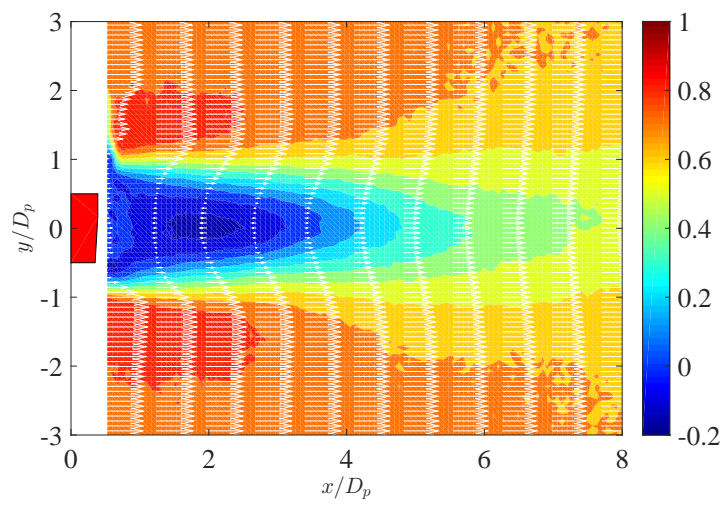

(b)

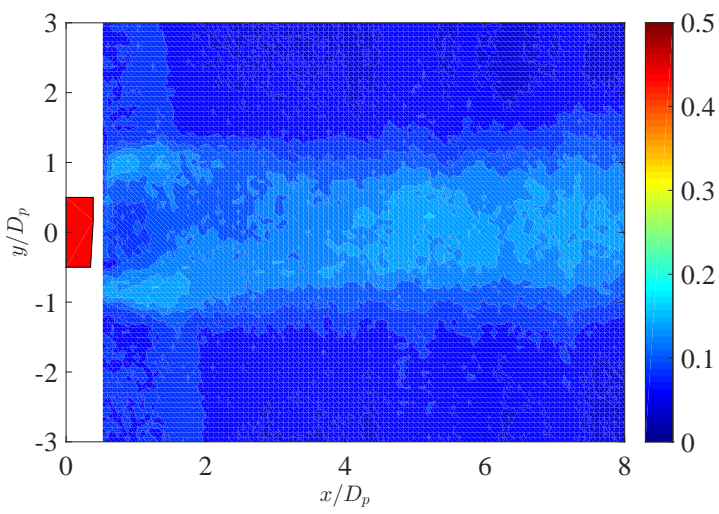

(d)

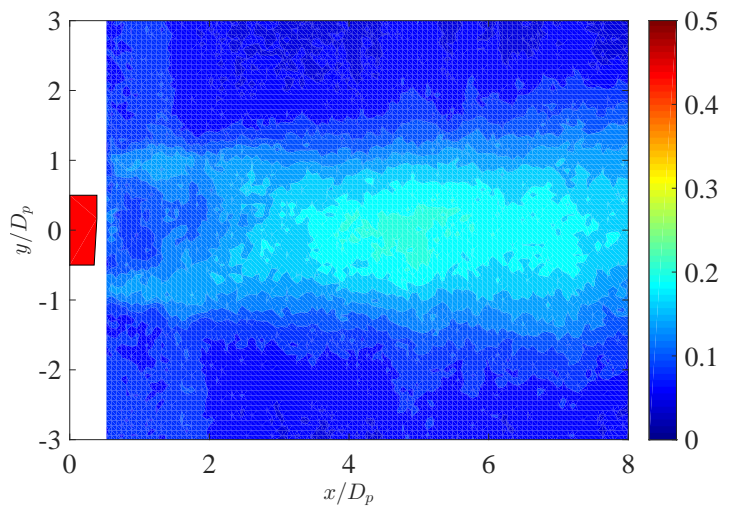

Fig. 14 Statistics in Case A at $z=11.25 \mathrm{~mm}:(a) U / U_{\infty}$ in the continuous mode; $(b) v_{\mathrm{rms}} / U_{\infty}$ in the continuous mode; (c) $U / U_{\infty}$ in the burst mode; $(d) v_{\text {rms }} / U_{\infty}$ in the burst mode. In the burst mode, $f_{\text {burst }} / f_{\text {shed }}=3.6$ and $\chi=0.5$.

the case of continuous mode. Although the velocity fluctuations are not as much suppressed as the optimal case of Case A, the profiles look closer to that of the high frequency case $\left(f_{\text {burst }} / f_{\text {shed }}=5.4\right)$ of Case A. The smaller amount of suppression of $v_{\text {rms }}$ in the far wake than that in Case $\mathrm{A}$ is also likely due to an additional disturbance. This argument is similar to Case A, but in Case B this additional disturbance may also be amplified through the interaction with the disturbance from the uncontrolled upper shear layer. From above, it can be concluded that the effect of vortex shedding suppression in the single-side configuration is greatly improved by adopting the burst mode. Although the suppression effect is less than that in the optimal case of double-side configuration, it can reach a level closer to the high frequency case of the double-side configuration.

\subsection{Differences in the flow control effect on the different cross sections}

Statistics at $z=11.25 \mathrm{~mm}$ (between two VG-PAs) in Cases A and B are illustrated in Figs. 14 and 15 . In the continuous mode of Case A (Figs. $14(a)$ and $(b)$ ), the wake in the vicinity of the pantograph head model is as wide as that in the uncontrolled case, but the downstream wake is similar to that at $z=0 \mathrm{~mm}$ (Fig. 10 ( $a$ ) (left)), and the fluctuations are suppressed similarly to those at $z=0 \mathrm{~mm}$ (Fig. $10(a)$ (right)). The suppression effects of the velocity fluctuations in the burst mode at $f_{\text {burst }} / f_{\text {shed }}=3.6$ and $\chi=0.5$ (Figs. $14(c)$ and $(d)$ ) are also similar to at $z=0$ mm (Figs. $12(a)$ and $(b)$ ).

In Case B, too, no remarkable differences in the distributions of $U$ and $v_{\text {rms }}$ are found between $z=0 \mathrm{~mm}$ and $z=11.25 \mathrm{~mm}$, as can be noticed from the comparisons between Figs. $15(a)$ and $(b)$ and Fig. $10(b)$ for the continuous mode and between Figs. $15(c)$ and $(d)$ and Figs. $13(a)$ and $(b)$ for the burst mode.

From above, it can be concluded that there is no significant spanwise variation in the downstream wake in terms of the distributions of $U$ and $v_{\mathrm{rms}}$. An exception is the region right behind the pantograph head model ( $\operatorname{say}, \mathrm{up}$ to $x / D_{p}=2$ ), in which the distributions are similar to those in the uncontrolled flow due to the absence of PA. The spanwise homogeneity observed in the downstream wake can be attributed to the fact that the vortical structure induced by the VG-PA has a cross-sectional velocity about $0.3 U_{\infty}$ with an advection velocity about $0.3 U_{\infty}$ (according to, e.g., Figs. $12(a)$ and $14(a)$ ). Namely, the streamwise length scale of cross-sectional mixing is about $D_{p}$, and the spanwise inhomogeneity due to the placement of VG-PAs is considered to have already been relaxed at $x / D_{p}=2$. 
(a)

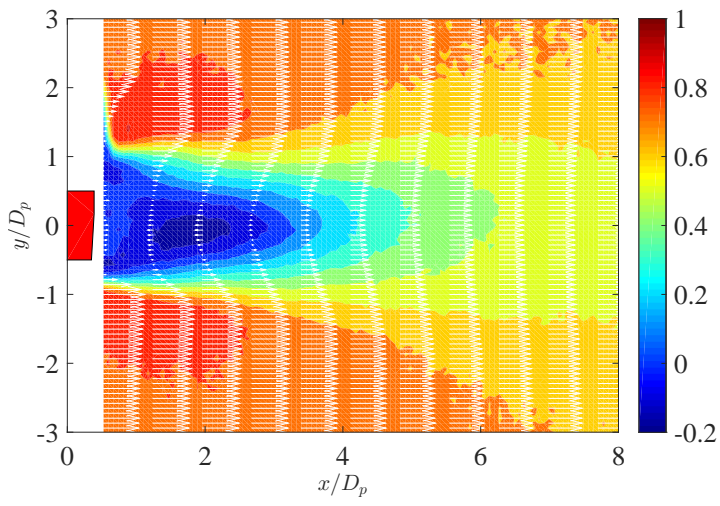

(c)

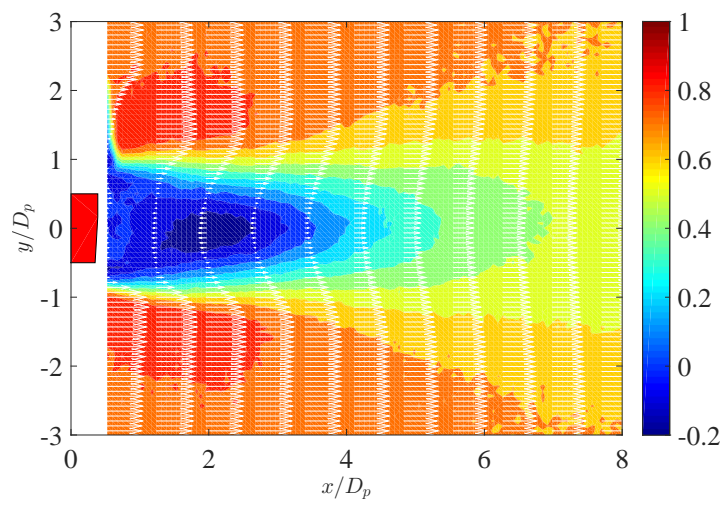

(b)

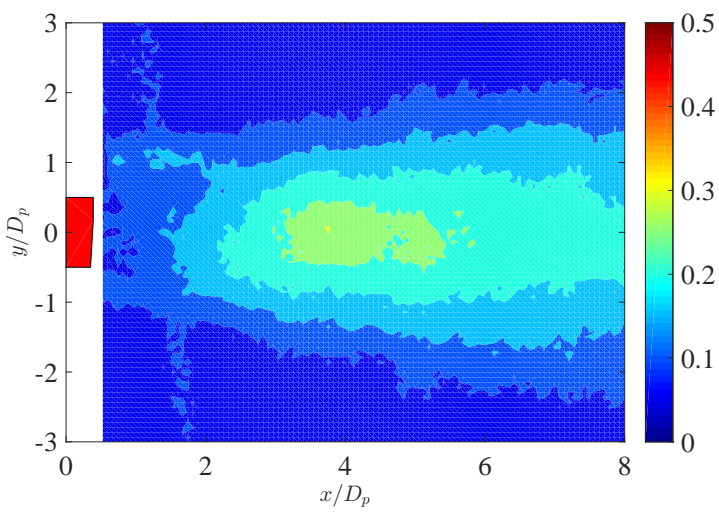

$(d)$

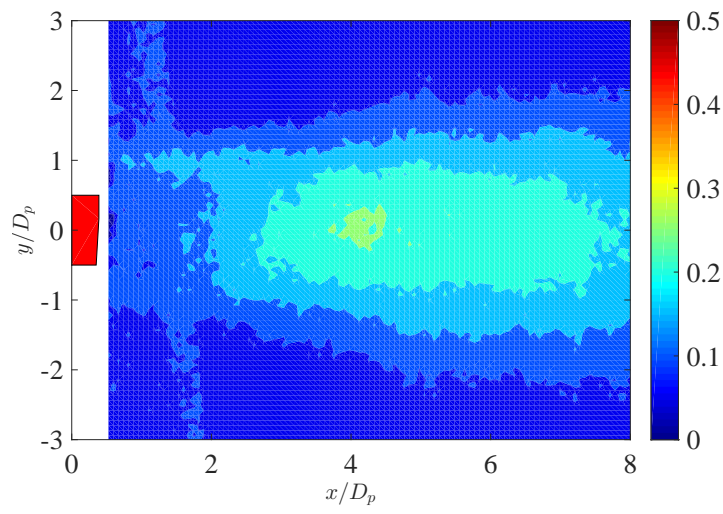

Fig. 15 Statistics in Case B at $z=11.25 \mathrm{~mm}:(a) U / U_{\infty}$ in the continuous mode; $(b) v_{\mathrm{rms}} / U_{\infty}$ in the continuous mode; $(c) U / U_{\infty}$ in the burst mode; $(d) v_{\text {rms }} / U_{\infty}$ in the burst mode. In the burst mode, $f_{\text {burst }} / f_{\text {shed }}=3.6$ and $\chi=0.5$.

\section{Conclusions}

Effects of vortex generator-type plasma actuators (VG-PAs) on the vortex shedding from a pantograph head model have been investigated by means of particle image velocimetry (PIV). The freestream velocity is $U_{\infty}=4.8 \mathrm{~m} / \mathrm{s}$; the Reynolds number based on the freestream velocity and the width of the pantograph head model is 7200 . The spanwise velocity induced by the VG-PA is $1.6 \mathrm{~m} / \mathrm{s}$, which is $33 \%$ of the freestream velocity.

In the continuous mode, the flow separation and the vortex shedding can significantly be suppressed when VGPAs are installed on the top and the bottom surfaces of the pantograph head (i.e., double-side configuration). From the comparison between two different spacings of VG-PAs, a greater effect is obtained when the spacing is set equal to the width of the pantograph head model. The maximum reduction rate of the root-mean-square (RMS) transverse velocity fluctuations is found to be about $60 \%$. When VG-PAs are installed on the bottom surface only (i.e., single-side configuration), in contrast, the vortex shedding is not much suppressed.

It has been demonstrated that the effect in the single-side configuration can be much improved by operating VG-PAs in the burst mode. The optimal burst frequency is found to be 3.6 times of the vortex shedding frequency, which resulted in about $30 \%$ reduction in the RMS transverse velocity fluctuations even in the single-side configuration. In addition, the position of the VG-PAs in the spanwise direction can have little effects on the flow control effects around the pantograph head.

The single-side configuration is preferable in practical applications, where the top surface of the pantograph head contacts with the overhead line. Although there are many issues left toward practical applications, e.g., investigation on the effects at the actual speed of bullet trains $(\simeq 100 \mathrm{~m} / \mathrm{s})$ and confirmation of the noise-reduction effect, the present study at least demonstrated an effective method which can also be used in the single-side configuration.

\section{Acknowledgments}

The authors are grateful to Drs. Shinnosuke Obi and Keita Ando (Keio University) and Dr. Takehisa Takaishi (JAXA) for fruitful discussion. The authors also appreciate Mr. Yuichi Sato (Railway Technical Research Institute, Japan: RTRI) 
and Mr. Satoru Hataoka (Keio University) for their extensive support on the wind-tunnel experiment at RTRI. This work was supported by a collaborative research program between Keio University and RTRI.

\section{References}

Asada, K. and Fujii, K., Burst frequency effect of DBD plasma actuator on the control of separated flow over an airfoil, Proceedings of the 6th AIAA Flow Control Conference (2012), AIAA Paper 2012-3054.

Bushnell, D. M., Longitudinal vortex control - Techniques and applications (The 32nd Lanchester lecture), Aeronautical Journal, Vol. 96, No. 958 (1992), pp. 293-312.

Corke, T. C., Enloe, C. L. and Wilkinson, S. P., Dielectric barrier discharge plasma actuators for flow control, Annual Review of Fluid Mechanics, Vol. 42 (2010), pp. 505-529.

Jukes, T. N. and Choi, K.-S., Flow control around a circular cylinder using pulsed dielectric barrier discharge surface plasma, Physics of Fluids, Vol. 21 (2009a), 084103.

Jukes, T. N. and Choi, K.-S., Control of unsteady flow separation over a circular cylinder using dielectric-barrier-discharge surface plasma, Physics of Fluids, Vol. 21 (2009b), 094106.

Jukes, T. N. and Choi, K.-S., Dielectric-barrier-discharge vortex generators: characterization and optimization for flow separation control, Experiments in Fluids, Vol. 52 (2012), pp. 329-345.

Jukes, T. N. and Choi, K.-S., On the formation of streamwise vortices by plasma vortex generators, Journal of Fluid Mechanics, Vol. 733 (2013), pp. 370-393.

Jukes, T. N., Segawa, T. and Furutani, H., Flow control on a NACA 4418 using dielectric-barrier-discharge vortex generators, AIAA Journal, Vol. 51 (2013), pp. 452-464.

Lin, J. C., Review of research on low-profile vortex generators to control boundary-layer separation, Progress in Aerospace Science, Vol. 38 (2002), pp. 389-420.

Lyn, D. A., Einav, S., Rodi, W., and Park J.-H., A laser-Doppler velocimetry study of ensenble-averaged characteristics of turbulent near wake of a square cylinder, Journal of Fluid Mechanics, Vol. 304 (1995), pp. 285-319.

Makino, T., Kakehi, Y., Iida, A., Terada, K. and Hattori, M., Noise characteristics of current collector for high-speed railway using delta-shaped collector head, JSME International Journal Series C, Vol. 41, (1998), pp. 338-346.

Mitsumoji, T., Sueki, T., Yamazaki, N., Sato, Y., Ikeda, M., Takinami, R., Gejima, H., and Fukagata K., Aerodynamic noise reduction of a pantograph panhead by applying a flow control method, Proceedings of the 11th International Workshop on Railway Noise, September 9-13, 2013, Uddevalla, Sweden (2013), Paper No. 068.

Moreau, E., Airflow control by non-thermal plasma actuators, Journal of Physics D: Applied Physics, Vol. 40 (2007), pp. 605-636.

Nati, G., Kotsonis, M., Ghaemi, S. and Scarano, F., Control of vortex shedding from a blunt trailing edge using plasma actuators, Experimental Thermal Fluid Science, Vol. 46 (2013), pp. 199-210.

Roth, J. R. and Dai, X., Optimization of the aerodynamic plasma actuator as an electrohydrodynamic (EHD) electrical device, Proceedings of the 44th AIAA Aerospace Sciences Meeting and Exhibit (2006), AIAA Paper 2006-1203.

Roy, S. and Wang, C.-C., Bulk flow modification with horseshoe and serpentine plasma actuators, Journal of Physics D: Applied Physics, Vol. 42 (2008), 032004.

Saha, A. K., Muralidhar, K., and Biswas, G., Experimental study of flow past a square cylinder at high Reynolds numbers, Experiments in Fluids, Vol. 29 (2000), pp. 553-563.

Sidorenko, A. A., Budovskiy, A. D., Maslov, A. A., Postnikov, B. V., Zanin, B. Y., Zverkov, I. D., and Kozlov, V. V., Plasma control of vortex flow on a delta wing at high angles of attack, Experiments in Fluids, Vol. 54 (2013), 1585.

Taylor, H. D., The elimination of diffuser separation by vortex generators, United Aircraft Corporation Report (1947), No. R-4012-3. 